\title{
Towards self-aware PerAda systems
}

\begin{abstract}
Pervasive Adaptation (PerAda) refers to massive-scale pervasive information and communication systems which are capable of autonomously adapting to highly dynamic and open technological and user contexts. PerAda systems are thus a special case of collective adaptive systems which have particular constraints e.g. they are networked and highly distributed; they involve interaction with humans; they are large scale; the boundaries of systems are fluid; their context is dynamic; and they operate using uncertain information. In order to achieve their ultimate goal of adapting seamlessly to their users and to deliver the expected quality of service at all times, we propose that these systems must exhibit self-awareness. This position statement proposes mechanisms by which self-awareness might be achieved.
\end{abstract}

\section{A Homunculus for PerAda Systems}

To be self-aware, a system must have an internal self-image: moreover, in the case of PerAda systems, that image must be dynamic as the system itself has a dynamic and fluid composition. Borrowing terminology used in both neurology and immunology we propose that collective systems must develop their own homunculus. Stemming from the Latin term "little man", the neurological homunculus refers to the functional map of the body represented in the brain which determines how it interacts with its environment; the immunological homunculus refers to the immune system's internal image of the body. Both the neurological and immunological homunculi can be viewed as a dynamic representation of the body and its various states: the representation is a reduced, virtual image of the system in that it contains far less information than the total amount of information contained in the body itself, but encodes the functional essence of the body's state [2. In contrast to traditional perceptions of immunity, the key concept of the homuculus is that auto-immunity, that is, the ability of the immune system to detect and respond to the state of the body's cells and tissues, is a normal and critical process of the functioning of the immune system; the image is created by body cells that impinge on the immune receptors and crucially by the response of cells to this information 3. This results in a self-referential system which has obvious advantages for achieving collective behaviour; it is not necessary for every part of the system to observe the state of the overall system but sufficient to observe how local parts of the system are behaving.

However, in contrast to the immunological or neurological homunculi, a pervasive system does not have a defined boundary. This fluidity of boundary requires the concept of self-awareness to be completely reviewed when self is a fluid entity. Thus, although the notion of internal-image of an engineered system has been previously considered in the literature, we suggest that in order to create 
a self-image of a PerAda system, we need to go beyond current thinking: we propose a PerAda homunculus might be created if the system can:

- Look inside: nodes and systems must be able to inspect and understand the implications of their own internal state and operational conditions

- Look outside: nodes and systems need to be aware of the environment in which they exist, including other nodes and systems as well as users of the environment

- Look inside from outside: nodes and systems need to be aware of how their actions and responses are perceived by other nodes and systems external to them, including users.

- Look backward: nodes and systems need to accumulate and make sense of information collected from the system in the past and draw correlations between data and actions

- Look forward: nodes and systems need to look forward in a predictive manner and anticipate potential problems and maybe take corrective action

We dub this new awareness look-* and propose that looking to the natural immune system and the mechanisms by which it computes a homunculus can provide some inspiration for building systems which encapsulate these concepts.

\section{The recursive nature of PerAda systems}

We further note that PerAda systems can be thought of as recursive, i.e. almost fractal in nature, in that a system consists of many subsystems, each of which can be thought of as system in itself such that each part is a reduced size copy of the whole with respect to the general challenges of adapting to the environment and in that the boundaries between the subsystems are fluid: in the horizontal dimension, at any time a system (component, devices, group, network etc.) may be joined by one or more other systems to form a new system incorporating the goals and context of all. Similarly, a system may split into two or more systems each requiring goals and context based on the parent system. In the vertical dimension a system might be considered to be at different levels depending on the viewpoint. Something that is a device from one point of view might be a component from another point of view. What we might view as a complete system (e.g. all the components, devices, groups and networks that exist within our car) might be seen as single component from another point of view (e.g. when considering the traffic flow through a city). Adaptation takes place across multiple scales; the adaptation process repeats itself from the small scale at the innermost level of the system (e.g. a component in a car) to the large scale outermost level (e.g. the traffic in a city). Again, looking to the immune system might inform our thinking: the immune system operates across significant different scales, ranging from interactions between tissues via cytokine release which occur across time-scales ranging from minutes to hours and distances of up to a metre to intercellular networks which operate across seconds over distances of a few millimetres [1]. 


\section{Conclusions}

In summary, collective systems are fluid, fractal-like systems which must develop their own internal self-image, a homunculus, through which they can successfully interact with the environment and collectively adapt. Steps towards defining and maintaining a collective homunculus will be made by considering self-awareness from a multi-faceted perspective, such as look-* self awareness which accounts for the self-referential nature of the homunculus and the fractal-like nature of the system itself.

\section{References}

1. R. Callard and J. Stark. Biological Networks, chapter Networks of the Immune System. World Scientific Publishing, 2007.

2. I. Cohen. Immune system computation and the immunological homunculus. In Model Driven Engineering Languages and Systems, pages 499-512. Springer, 2006.

3. I. Cohen. Real and artificial immune systems; computing the state of the body. Nature Reviews Immunology, 2007. 